\title{
Study of Linearly Chirped 1-Dimensional Bragg Gratings Using Confluent Hypergeometric Function
}

\author{
A. Bahtiar* and R. E. Siregar \\ Department of Physics, FMIPA, Universitas Padjadjaran \\ Jatinangor, Sumedang 45363, West Java
}

\begin{abstract}
A finite one-dimensional, linearly Bragg grating with chirp parameter $\mathrm{C}$ and index modulation depth $\mathrm{n}_{1}$ or coupling constant $\kappa \propto \mathrm{n}_{1}$ is considered for numerical analysis of its performances by converting its coupled equations into a confluent hypergeometric equation. The operational parameters considered for the analysis consist of the group delay, the reflectance (R), the reflectance ripple (RR), the reflectance bandwidth (BW) and the group delay ripple (GDR). It is shown that increasing $\mathrm{C}$ leads to decreasing GDR and increasing BW which are favorable to the device performance. However, the RR is relatively unaffected while R suffers from an undesirable diminution. On the other hand, increasing $\kappa$ results in the enhancement of $\mathrm{R}$ and $\mathrm{BW}$ while reducing RR when $\kappa$ is increased beyond a certain turning point. But these are attained at the expense on enlarged GDR. The results of this study clearly points to the need of compromising between $\mathrm{C}$ and $\kappa$ for the optimal operation of the device.
\end{abstract}

KEYWORDS: chromatic dispersion, chirped fiber Bragg grating.

\section{INTRODUCTION}

Since the successful application of erbium-doped amplifiers, the only major factor limiting the performance of highspeed optical-fiber communication systems is its optical chromatic dispersion responsible for the potentially errors generating pulse broadening effect. Dispersion compensating fiber is currently used as the standard solution for the chromatic dispersion compensation.

Various schemes for dispersion compensation have been proposed since the late nineteen seventies and early nineteen eighties [1]. Most of these proposals are nevertheless either unsuitable for on-line implementation or short of meeting the cost-effective criterion for their applications. In a later study, Ouelette [2] explored further the advantage of early works on linearly chirped Bragg grating [3-5] which was known to allow large dispersion effects of either sign when operated in the reflection mode. The dispersion compensation module consists of a circulator and a fiber Bragg grating, as depicted in Fig. 1. This module works as a signal repeater in a conventional optical-fiber communication systems .

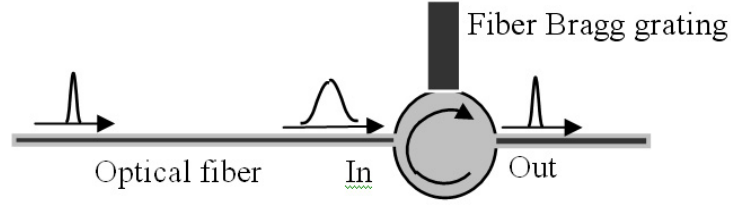

FIG. 1: The idea of a dispersion compensator.

${ }^{*}$ E-MAIL: rustameunpad.ac.id
However, this scheme was invariably plagued by the undesired irregular oscillatory behavior or simply ripple of the dispersion effect which will lead to power penalty and uncontrollable compensation effect in the system. Oulette [2] argued that this detrimental behavior was mainly due to the presence of side lobes in the reflectivity of the unchirped grating, giving rise to wave coupling at the side lobes frequencies outside the desired resonance region. He went on to show that this effect could be minimized by decreasing the coupling coefficient gradually toward the ends of the coupling region. It was also demonstrated in a more recent experimental study [6] that apodization of the linearly chirped grating leads to reduce side lobes and hence the reduction of unwanted in the dispersion delay ripple as well.

Despite advances achieved so far on both recent experimental and theoretical studies as briefly described above, exact analytical result on dispersion/group delay characteristics are relatively short in supply. It was only recently that Belai et. al. [7] succeeded in driving simple analytical expressions for reflection coefficient and group delay of a linearly chirped Bragg grating employing the confluent hypergeometric functions at their asymptotic limit. It was found in their analysis that in order to comply with the needs of suppressing dispersion delay ripples as well as maintaining high reflectivity, both the chirp parameter and index modulation depth must be made as large as possible, leading to possibly unrealistic demand on the refractive index.

We report in this paper a detailed numerical investigation of the effects of chirp parameter and refractive index modulation depth of a linearly chirped one-dimensional grating. The basic formulation of Belai et. al [7] will be adopted here without submitting ourselves to the asymptotic condition. It was shown that the results of this work complements to the result of Ref. [7] in the optimization of the system parameters for the best performance specified in terms of high reflectance 
with small ripple, minimum ripple in group delay and wide operational bandwidth.

\section{THE MODEL}

The structure of one-dimensional chirped fiber Bragg grating is sketched in Figure 2, indicating the propagation of the forward and backward waves along the $\mathrm{z}$-axe. The refractive index of the chirped grating is given by

$$
n(z)=n_{\circ}+n_{1} \cos [G(z) z]
$$

where $n_{\circ}$ is the fixed background refractive index while $n_{1}$ is a parameter denoting the modulation depth with $\mathrm{n}_{1} / \mathrm{n}_{\circ}<<$ 1 representing a shallow grating, and $\mathrm{G}(\mathrm{z})$ denoting the local spatial frequency of the system.

The linear chirp considered in this work is specified by the following expression for $\mathrm{G}(\mathrm{z})$,

$$
G(z)=G_{\circ}+C z
$$

Here $G_{\circ}$ is the center of the spatial frequency and $C$ is the chirp parameter which is held constant within the grating ($\mathrm{L} / 2 \leq \mathrm{z} \leq \mathrm{L} / 2$ ) and is equal to zero outside the grating.

\section{COUPLED-MODE EQUATIONS AND THEIR SOLUTION}

The steady state electric field $\mathrm{E}(\mathrm{z})$ in the grating satisfies the Helmholtz equation for the case of shallow grating, namely

$$
\frac{d^{2} E(z)}{d z^{2}}+\frac{\omega^{2}}{c^{2}} n^{2}(z) E(z)=0
$$

Expressing the electric field as a superposition of the counter propagating waves,

$$
E(z)=A(z) e^{i k z}+B(z) e^{-i k z}
$$

If $\mathrm{A}(\mathrm{z})$ and $\mathrm{Bz}$ are slow varying amplitudes, and $\mathrm{k}=\mathrm{n}_{\circ} \omega / \mathrm{c}$ is close around $G_{\circ} / 2$, substitution of eq. (4) into eq. (3) results in the following coupled-mode equations,

$$
\begin{aligned}
& \frac{d A}{d z}-i \delta A=i \kappa B e^{-i \phi} \\
& \frac{d B}{d z}+i \delta B=-i \kappa A e^{i \phi}
\end{aligned}
$$

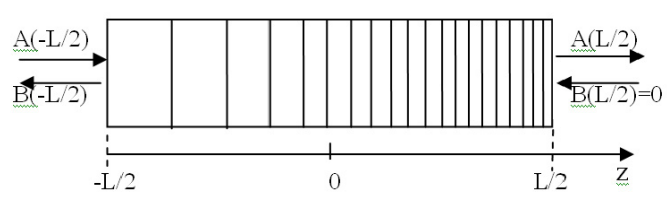

FIG. 2: Sketch of a chirped fiber Bragg grating of total length L.
In those equations the frequency detuning $\delta$ is given by

$$
\delta=\frac{\omega}{c} n_{\circ}-\frac{G_{\circ}}{2}=\frac{n_{\circ}}{c}\left(\omega-\omega_{B}\right)
$$

where $\omega_{B}=\mathrm{cG}_{\circ} /\left(2 \mathrm{n}_{\circ}\right)$ is the Bragg angular frequency at the center of the grating. Further, the coupling constant $\kappa$ is

$$
\kappa=G_{\circ} \frac{n_{1}}{2 n_{\circ}}
$$

while

$$
\phi(z)=\left[G(z)-G_{\circ}\right] z=C z^{2}
$$

Now we consider,

$$
\begin{gathered}
A(z)=a(z) e^{i \delta z} \\
B(z)=b(z) e^{-i \delta z}
\end{gathered}
$$

then the coupled-mode equations in eq. (5) changes to be

$$
\begin{aligned}
& \frac{d a}{d z}=i \kappa b e^{-i\left(2 \delta z-C z^{2}\right)} \\
& \frac{d b}{d z}=-i \kappa a e^{i\left(2 \delta z-C z^{2}\right)}
\end{aligned}
$$

Using eq.(10) we get the following second order differential equations,

$$
\begin{aligned}
& \frac{d^{2} a}{d z^{2}}-i 2 C(z-q) \frac{d a}{d z}-\kappa^{2} a=0 \\
& \frac{d^{2} b}{d z^{2}}-i 2 C(z-q) \frac{d b}{d z}+\kappa^{2} b=0
\end{aligned}
$$

where

$$
q=\frac{\delta}{C}
$$

By substitution of

$$
x=i C(z-q)^{2}
$$

into eq. (11) we have the following second order differential equations,

$$
\begin{aligned}
& x \frac{d^{2} a}{d x^{2}}+\left(\frac{1}{2}-x\right) \frac{d a}{d x}+i \gamma a=0 \\
& x \frac{d^{2} b}{d x^{2}}+\left(\frac{1}{2}-x\right) \frac{d b}{d x}-i \gamma b=0
\end{aligned}
$$

where

$$
\gamma=\frac{\kappa^{2}}{4 C}
$$

Eq's. (14) are known as the Confluent Hypergeometric Differential Equations, and their solutions are [2, 4]

$$
\begin{array}{r}
a(z)=P_{1} u_{1}(z)+P_{2} u_{2}(z) \\
b(z)=Q_{1} U_{1}^{*}(z)+Q_{2} u_{2}^{*}(z)
\end{array}
$$


Here, P's and Q's are constants and

$$
\begin{array}{r}
u_{1}(z)=F\left(-i \gamma ; \frac{1}{2} ; i C(z-q)^{2}\right) \\
u_{2}(z)=(z-q) F\left(\frac{1}{2}-i \gamma ; \frac{3}{2} ; i C(z-q)^{2}\right)
\end{array}
$$

and the confluent hypergeometric function $\mathrm{F}(\mathrm{a} ; \mathrm{c} ; \mathrm{x})$ has the following form [8 ],

$$
\begin{aligned}
F(a ; c ; x) & =1+\frac{a}{c} \frac{x}{1 !}+\frac{a(a+1)}{c(c+1)} \frac{x^{2}}{2 !} \\
& +\frac{a(a+1)(a+2)}{c(c+1)(c+2)} \frac{x^{3}}{3 !}+\cdots
\end{aligned}
$$

From eq.(9) and eq. (16) we have the coefficient of reflectivity at $\mathrm{z}=-\mathrm{L} / 2$ as

$$
\begin{aligned}
r\left(-\frac{1}{2} L, \delta\right) & =\frac{b\left(-\frac{1}{2} L\right)}{a\left(-\frac{1}{2} L\right)} e^{i \delta L} \\
& =\frac{\frac{Q_{1}}{P_{2}} u_{1}^{*}\left(-\frac{1}{2} L\right)+\frac{Q_{2}}{P_{2}} u_{2}^{*}\left(-\frac{1}{2} L\right)}{\frac{P_{1}}{P_{2}} u_{1}\left(-\frac{1}{2} L\right)+u_{2}\left(-\frac{1}{2} L\right)} e^{i \delta L}
\end{aligned}
$$

The constants P's and Q's are determined as follows:

(i) For $\mathrm{z} \approx \mathrm{q}$, eq's. (17) and (18) give $\mathrm{u}_{1}(\mathrm{z}) \approx 1$, and $\mathrm{u}_{2}(\mathrm{z}) \approx(\mathrm{z}-$ q), so that

$$
\begin{gathered}
a(z)=P_{1}+P_{2}(z-q)+O(z-q)^{2} \\
b(z)=Q_{1}+Q_{2}(z-q)+O(z-q)^{2}
\end{gathered}
$$

Then, by using eq. (10) we get

$$
\begin{aligned}
& P_{2} \approx i \kappa Q_{1} e^{-i\left(2 \delta z-C z^{2}\right)} \\
& Q_{2} \approx-i \kappa P_{1} e^{i\left(2 \delta z-C z^{2}\right)}
\end{aligned}
$$

(ii) Because of $\mathrm{b}(\mathrm{L} / 2)=0$, eq.(16) gives $Q_{1} u_{1}^{*}(L / 2)+$ $Q_{2} u_{2}^{*}(L / 2)=0$, or

$$
\frac{Q_{2}}{Q_{1}}=-\frac{u_{1}^{*}\left(\frac{1}{2} L\right)}{u_{2}^{*}\left(\frac{1}{2} L\right)}
$$

Then from eq. (20) and (21) we get

$$
\frac{P_{1}}{P_{2}}=\frac{Q_{2}}{\kappa^{2} Q_{1}}=-\frac{1}{\kappa^{2}} \frac{u_{1}^{*}\left(\frac{1}{2} L\right)}{u_{2}^{*}\left(\frac{1}{2} L\right)}
$$

Eq.'s (20), (21) and (22) give us

$$
\begin{array}{r}
\frac{Q_{1}}{P_{2}}=\frac{1}{\kappa^{2}} \frac{Q_{2}}{P_{1}}=-i \frac{1}{\kappa} e^{j\left(2 \delta z-C z^{2}\right)} \\
\frac{Q_{2}}{P_{2}}=\frac{Q_{2}}{P_{1}} \times \frac{P_{1}}{P_{2}}=i e^{i\left(2 \delta z-C z^{2}\right)} \frac{u_{1}^{*}\left(\frac{1}{2} L\right)}{\kappa u_{2}^{*}\left(\frac{1}{2} L\right)}
\end{array}
$$

Using eq. (16) for $\mathrm{z}=-\mathrm{L} / 2$, and eq's (22), (23) and (24) we have the following coefficient reflection

$$
r\left(-\frac{1}{2} L, \delta\right)=\frac{i}{\kappa} e^{-i C L^{2} / 4}\left[\frac{-u_{1}^{*}\left(-\frac{1}{2} L\right) u_{2}^{*}\left(\frac{1}{2} L\right)+u_{1}^{*}\left(\frac{1}{2} L\right) u_{2}^{*}\left(-\frac{1}{2} L\right)}{-\frac{1}{\kappa^{2}} u_{1}^{*}\left(\frac{1}{2} L\right) u_{1}\left(-\frac{1}{2} L\right)+u_{2}^{*}\left(\frac{1}{2} L\right) u_{2}\left(-\frac{1}{2} L\right)}\right]
$$

The phase of the output $\mathrm{B}(-\mathrm{L} / 2)$ can be obtained relatively to input $\mathrm{A}(-\mathrm{L} / 2)$ as

$$
\varphi=\tan ^{-1} \frac{\operatorname{Im}(r)}{\operatorname{Re}(r)}
$$

while the group delay is expressed as

$$
\tau=-\frac{\partial \varphi}{\partial \omega}=-\frac{n_{\circ}}{c} \frac{\partial \varphi}{\partial \delta}
$$

Finally, the reflectance of the grating is given by

$$
R\left(-\frac{1}{2} L, \delta\right)=\left|r\left(-\frac{1}{2} L, \delta\right)\right|^{2}
$$

\section{RESULTS AND DISCUSSION}

The grating length $\mathrm{L}$ in this work is $10 \mathrm{~mm}$ and the background refractive index $\mathrm{n}_{\circ}$ is 1.5 . The center of the operational wavelength $\lambda_{B}$ is $1550 \mathrm{~nm}\left(\mathrm{f}_{\mathrm{o}}=193.55 \mathrm{THz}\right)$ and the Bragg spatial periodicity at the center of grating is $\Lambda_{B}=\lambda_{B} / 2 \mathrm{n}_{\circ}=517 \mathrm{~nm}$, while the center of the spatial frequency $\mathrm{G}_{o}=2 \pi / \Lambda_{B}=12.147 \times 10^{3} \mathrm{~mm}^{-1}$. From eq. (7), the value of coupling parameter $\kappa=1 \mathrm{~mm}^{-1}$ corresponds to the modulation depth of refractive index $\mathrm{n}_{1} / \mathrm{n}_{o}=1.65 \times 10^{-4}$. By use of eq. (6), the relationship between $\lambda$ and $\delta$ is

$$
\lambda \approx \lambda_{B}\left(1-\frac{\lambda_{B} \delta}{2 \pi n_{\circ}}\right)
$$

Using eq. (29) then $\delta=1 / \mathrm{mm}$ corresponds to $\lambda-\lambda_{B}=-0.255$ $\mathrm{nm}$. Using eq. (27), the group delay can be formulated as

$$
\tau(p s)=5 \frac{\partial \varphi(r a d)}{\partial \delta\left(m^{-1}\right)}
$$

The definition of the reflectance $(\mathrm{R})$, the reflectance ripple (RR), the reflectance bandwidth (BW), and the group delay ripple (GDR) used in this work are shown in Figure 3, where 


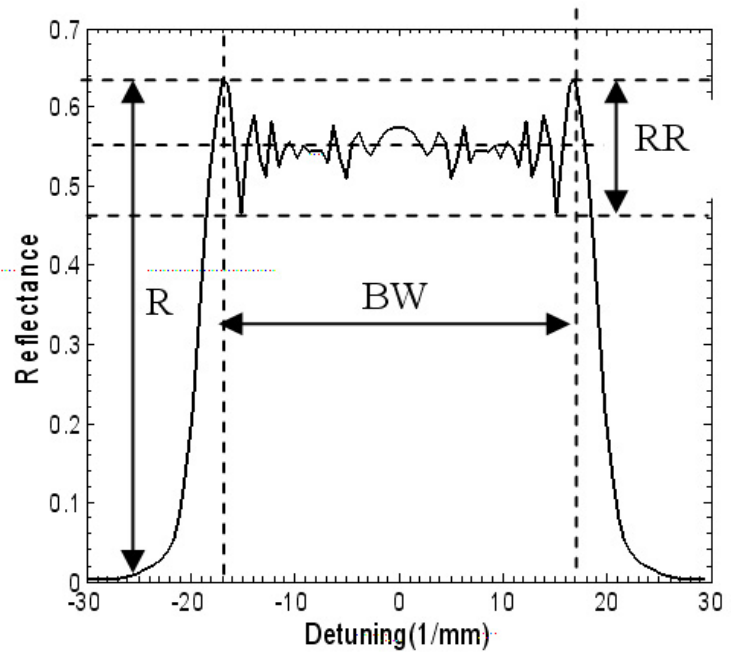

(a)

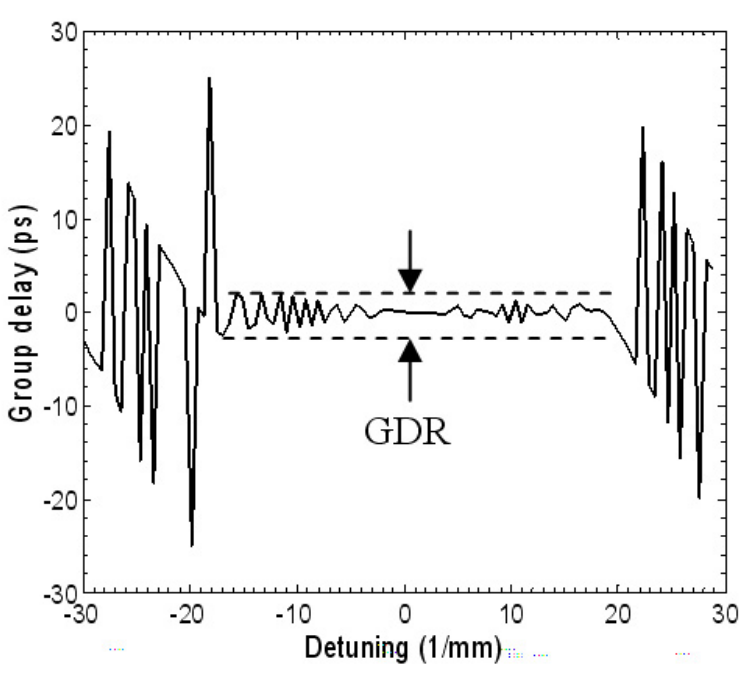

(b)

FIG. 3: The definition of reflectance (R), the reflectance ripple (RR), the reflectance bandwidth (BW), and the group delay ripple (GDR) in this work.

$\mathrm{R}$ is the maximum value of reflectance, $\mathrm{RR}$ is the maximum value of the peak to peak of the ripple, BW is the bandwidth of the reflectance at $50 \%$ of maximum reflectance and GDR is the maximum peak to peak of group delay.

The $\kappa$ dependence of $\mathrm{R}$ for various values of the chirp parameter $\mathrm{C}$ is shown in Figure 4(a). We see that for each value of $\mathrm{C}$ the maximum values of reflectance $(\mathrm{R})$ are higher for the higher value of $\kappa$, and at each value of $\kappa$, the values of $\mathrm{R}$ are higher for the lower value of $\mathrm{C}$. From the curves in that figure we can expressed the $\kappa$ and $\mathrm{C}$ dependence of $\mathrm{R}$ as

$$
R=1-\exp \left(-\frac{a \kappa^{b_{1}}}{C^{b_{2}}}\right)
$$

where $a=2 \mathrm{~mm}, \mathrm{~b}_{1}=2$ and $\mathrm{b}_{2}=0.5$ will fit the curves.

The reflectance ripple (RR) in Figure 3(b) has the maximum value around the coupling parameter $\kappa=1.05 \mathrm{~mm}^{-1}$; the coupling parameter reduces RR at $\kappa>1.05 \mathrm{~mm}^{-1}$. From Figure 4 (a) and (b) we find that increasing [106b] beyond a certain turning point will result in the higher $\mathrm{R}$ and lower RR. These characteristic are very important in designing the device.

The $\kappa$ dependence of BW for various values of the chirp parameter $\mathrm{C}$ is shown in Figure 4(c). We see that the bandwidth $\mathrm{BW}$ is a linear function of the chirp parameter $\mathrm{C}$. We can see also that at a given value of $\kappa$, the values of $\mathrm{BW}$ are higher for the higher the values of $\mathrm{C}$. From the curves in this figure, we can express $\mathrm{BW}$ as a function of $\kappa$ and $\mathrm{C}$ as follows:

$$
B W=2 C+0.5 \kappa
$$

In Figure 4(d), we see that for each value of C, the values of GDR are higher for the higher value of $\kappa$; but at each value of $\kappa$, the values of GDR are lower for higher values of C. From the curves in this figure, we can express GDR as a function of $\kappa$ and $\mathrm{C}$ as follows:

$$
G D R=\alpha C+\frac{\beta \kappa}{C}
$$

where $\alpha=0.25 \mathrm{ps} \mathrm{mm}^{2}$, and $\beta=15 \mathrm{ps} \mathrm{mm}$.

From eqs. (32) and (33) we find the good relationship between BW and GDR at each value of $\kappa$. However, the $\mathrm{C}$ dependence of BW and GDR are in contradiction with reflectance R. A good device for dispersion compensation has characteristics with a high reflectance, a wide bandwidth, a low reflectance ripple and a low group delay ripple. From the above results, we have to make compromising values between coupling and chirp parameters.

\section{CONCLUSION}

It is shown that increasing $\mathrm{C}$ leads to decreasing GDR and increasing $\mathrm{BW}$, which are favorable to the device performance. However, the RR is relatively unaffected while $\mathrm{R}$ suffers from an undesirable diminution. On the other hand, increasing $\kappa$ results in the enhancement of $\mathrm{R}$ and $\mathrm{BW}$ while reducing $\mathrm{RR}$ when $\kappa$ is increased beyond a certain turning point. But these are attained at the expense on enlarged GDR. The results of this study clearly points to the need of compromising between $\mathrm{C}$ and $\kappa$ for the optimal operation of the device.

\section{Acknowledgments}

The authors wish to thank Prof. M. O. Tjia of ITB for many valuable discussions and useful contributions. 


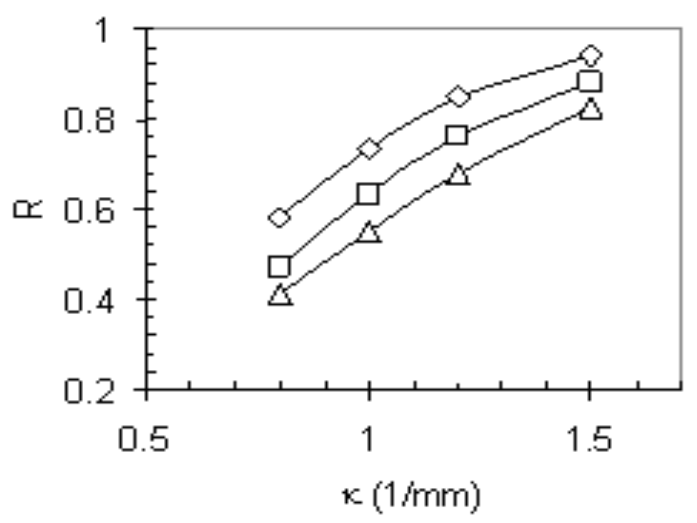

(a)

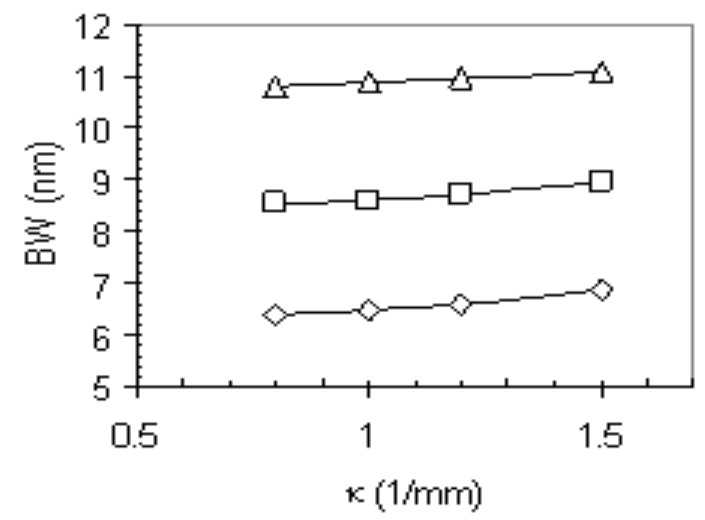

(c)

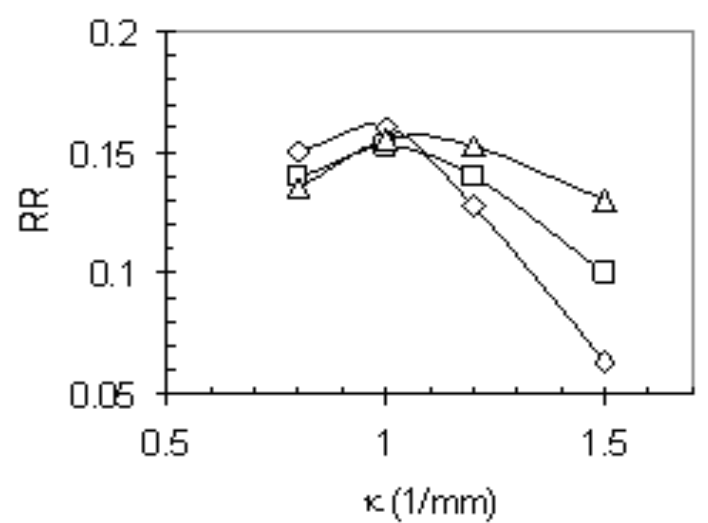

(b)

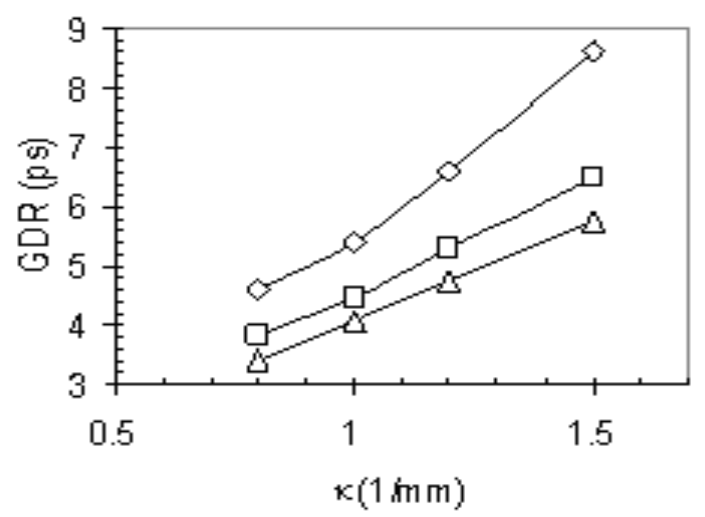

(d)

FIG. 4: The $\kappa$ dependence of R, RR, BW and GDR for various values of $\mathrm{C} ; \mathrm{C}=3 \mathrm{~mm}^{-2}(\diamond), \mathrm{C}=4 \mathrm{~mm}^{-2}(\square), \mathrm{C}=5 \mathrm{~mm}^{-2}(\Delta)$

[1] R. W. Hunsperger, Integrated Optics: Theory and Technology (Springer-Verlag, Berlin, p. 100, 1984).

[2] F. Ouelette, Optics Lett. 12, 847 (1987).

[3] M. Matsuhara, K. O. Hill, and A. Watanabe, J. Opt. Soc. Am. 65, 804 (1975).

[4] H. Kogelnik, Bell Syst. Tech. J. 55, 109 (1976).

[5] M. Matsuhara and K. O. Hill, Appl. Opt.,13, 1853-1856 (1974).

[6] K. O. Hill, F. Bilodeau, B.Malo, T. Kitagawa, S. Thriault, D. C. Johnson and J. Albert, Optics Lett. 19, 1314 (1994).
[7] O.V. Belai, E.V. Podivilov, D. A. Shapiro, Group delay in Bragg grating with linear chirp, arXiv: physics/0604091 v1 11 April 2006.

[8] M. Abramowitz et al. (Eds.), "Confluent Hypergeometric Functions." Ch. 13 in Handbook of Mathematical Function with Formulas, Graph, and Mathematical Tables (New York: Dover, pp. 503-515, 1972). 